\title{
Pure Hemi-Chorea Resulting from an Acute Phase of Contralateral Thalamic Lacunar Infarction: A Case Report
}

\author{
Teruyuki Takahashi $^{\mathrm{a}}$ Hideaki Kanamori $^{\mathrm{b}, \mathrm{c}}$ \\ Rihiro Shigehara ${ }^{\text {c c }}$ Sachiko Nonaka Takahashi ${ }^{\text {b }}$ \\ Masato Tamura ${ }^{d}$ Toshiaki Takasu $^{d}$ \\ Marohito Murakami ${ }^{\mathrm{b}, \mathrm{c}}$ \\ Departments of ${ }^{a}$ Neurology and ${ }^{b}$ Internal Medicine, Hino Municipal Hospital, \\ 'Department of Internal Medicine, School of Medicine, Keio University, and \\ ${ }^{\mathrm{d} D e p a r t m e n t}$ of Neurology, School of Medicine, Nihon University, Tokyo, Japan
}

\section{Key Words}

Pure hemi-chorea - Thalamic lacunar infarction · Diffusion-weighted image · DWI ·

Single-photon emission computed tomography $\cdot$ SPECT

\begin{abstract}
Background: Thalamic lesions give rise to a variety of clinical syndromes such as pure sensory stroke, ataxic hemiparesis, and rarely involuntary movements including chorea. Generally and classically, lacunar infarction in the subthalamic nucleus has been regarded as the lesion mainly responsible for hemi-chorea and hemi-ballismus, on the basis of previous anatomical studies.

Case Presentation: This report describes the case of an 81-year-old man who developed sudden-onset pure hemi-chorea in the right limbs resulting from an acute phase of left thalamic lacunar infarction detected on a diffusion-weighted image (DWI) in an MRI study. The patient had no other neurological symptoms such as ataxic hemiparesis and sensory disturbance. A single-photon emission computed tomography (SPECT) study using the ${ }^{99 m}$ TcECD Patlak plot method demonstrated significant perfusional asymmetry between the right and left thalami $(p=0.0035)$, consistent with the left thalamic lesion on DWI.
\end{abstract}

Conclusion: It is speculated that this perfusional asymmetry, in particular the hypoperfusion in the left thalamus, detected by SPECT might play the most important role in the contralateral pure hemi-chorea as a rare neurological manifestation in this case. 


\section{Background}

Thalamic lacunar infarction resulting from arteriosclerotic thrombosis of a single penetrating artery gives rise to a variety of clinical syndromes such as pure sensory stroke, ataxic hemiparesis, painful ataxic hemiparesis, hypesthetic ataxic hemiparesis and rarely involuntary movements including tremor, asterixis, athetosis, dystonia, pseudochoreoathetosis and chorea [1-9]. In many previous reports, various forms of hyperkinetic or hypokinetic disorders have been described following focal cerebrovascular lesions [1-12]. However, involuntary movement disorders are very uncommon clinical manifestations in the acute phase of ischemic stroke, and particularly hemi-chorea is regarded as one of the rarest neurological symptoms [115]. Therefore, the mechanism responsible for hemi-chorea following a cerebrovascular lesion is not yet completely understood [1-15].

This report describes the case of an 81-year-old man who developed sudden-onset pure hemi-chorea in the right limbs resulting from an acute phase of left thalamic lacunar infarction. The patient had no other neurological symptoms. This report includes a discussion of the anatomical basis for the clinico-radiological correlation and the pathophysiological mechanism of this rare condition, referring to previous literature [1-15].

\section{Case Presentation}

An 81-year-old right-handed man with a history of hypertension was admitted to our hospital because of involuntary movements in his right arm and leg, which had suddenly developed 5 days before admission. His medical and familial histories were unremarkable, except for a surgical operation for a gallbladder stone at the age of 64 .

On neurological examination, the patient had no visual field deficit and a full range of eye movement without nystagmus. He had no facial weakness. On manual motor tests, muscle strength and tone were almost normal in all limbs. Deep tendon reflexes were symmetrically brisk, and plantar responses were flexor. Sensory evaluations including pinprick, vibratory and joint position sense were almost normal in his face, on the trunk and four limbs. Other sensory disturbances including hypo- and hyperesthesia were not observed. He demonstrated prominent choreiform movements in the distal part of his right limbs. His right arm was affected more severely than his leg, but his face was spared. Involuntary movements were present at rest and exacerbated during voluntary actions. In addition, he could not suppress the involuntary movements by mental concentration. The fine finger movements of his right hand were clumsy due to the involuntary movements; therefore, cerebellar function tests were difficult to evaluate. While finger-to-nose testing was performed with his left hand, choreiform movements were noted in his right hand. Owing to right hemi-chorea, he tilted to the right when standing, and he was unable to walk without assistance as a result of severe gait instability. The neuropsychological examination showed an almost normal overall cognitive state (Mini-Mental State Examination score $=22$ ) with a mild decline in abstractive and constructive abilities.

His blood pressure was $184 / 108 \mathrm{~mm} \mathrm{Hg}$ on admission. The results of initial routine laboratory examinations were almost normal, except for slight anemia. His serum glucose level was $83 \mathrm{mg} / \mathrm{dl}$ and $\mathrm{HbA1c}$ was $4.7 \%$. The blood examinations for connective tissue diseases such as rheumatoid factor and antinuclear, anticardiolipin, antiphospholipid and anti-double-strand-DNA antibodies, Wilson's disease, vitamin E deficiency and acanthocytosis were all negative or unremarkable. Plaques at the origin of both internal carotid arteries, with stenosis of $30 \%$ on the right and $40 \%$ on the left, were demonstrated by echo Doppler examination. Surface electromyography (sEMG) recordings were performed to evaluate his involuntary movements at the distal part of both upper limbs at rest ( fig. $_{\text {. }}$ 1a) and under the calculation task (fig. 1b). The sEMG recordings demonstrated several synchronized 
grouping discharges in the right musculus brachioradialis and musculus extensor carpi ulnaris. These grouping discharges appeared irregularly and had a relatively long duration ( $>1 \mathrm{~s})$. In addition, the frequency and amplitude of the grouping discharges increased remarkably under the calculation task. These sEMG features were consistent with typical choreiform movements. In an initial brain magnetic resonance imaging (MRI) study (1.5 tesla), diffusion-weighted images (DWI) revealed a small lesion with high signal intensity at the left thalamus suggesting an acute phase of lacunar infarction (fig. 2a). There was no hyperintense lesion at the striatum, subthalamic nucleus (STN) or globus pallidus (GP) on simultaneous DWI. No significant intracranial vascular abnormality was observed on MR angiography.

After admission, the patient immediately received anti-platelet therapy (sodium ozagrel at 160 $\mathrm{mg} /$ day and edaravone at $60 \mathrm{mg} /$ day) for the acute left thalamic lacunar infarction. Moreover, for the involuntary movements in his right limbs, haloperidol was administered at $0.75 \mathrm{mg} / \mathrm{day}$. As a result of appropriate treatment, his right hemi-chorea improved gradually. On follow-up brain MRI study performed in the post-acute phase on day 14 after admission, his left thalamic lesion had disappeared on DWI, but it remained as a chronic lesion on T2-WI (fig. 2b). In addition, the simultaneous T2-WI revealed diffuse and pale lesions with high signal intensity in the bilateral basal ganglia and remarkable brain atrophy in the bilateral frontotemporal lobes, suggesting chronic deficiency of cerebral blood flow (CBF) (fig. 2b). A single-photon emission computed tomography (SPECT) study using the $99 \mathrm{~m}$ Tc-ECD Patlak plot method was performed on day 18, at the time when his right hemichorea had completely disappeared. On SPECT study (fig. 2c), a prominent perfusional asymmetry was observed between the right and left thalami. The regional (r) CBF in the thalamus, quantified by a fully automated analysis software, 'FineSRT' [mean \pm standard deviation (SD) in milliliters per 100 grams per minute], significantly decreased by about $43.7 \%(p=0.0035)$ in the left $(23.57 \pm 1.02)$ compared with the right $(41.85 \pm 0.78)$. On the other hand, a relative increase of $\mathrm{rCBF}$ was revealed in the left head of the caudate nucleus $(45.23 \pm 0.69)$ compared with that in the right head $(39.56 \pm 0.36)$ or the left thalamus. A diffuse decline of rCBF in the bilateral frontal cortexes was observed. The patient was discharged on day 24 .

\section{Discussion}

Thalamic lesions have been associated with various forms of involuntary movement disorders including focal dystonia, hemi-dystonia, tremor, asterixis, athetosis, ballismus, pseudochoreoathetosis and chorea [1-9]. In general, these involuntary movement disorders after ischemic stroke are regarded as really uncommon neurological manifestations [1-15]. D'Olhaberriague et al. [13] reported that in their study 9 patients with movement disorders were identified among 908 ischemic stroke cases (1\%). In addition, Arboix et al. [14] reported on 39 patients with atypical lacunar syndrome including movement disorders who accounted for $2.1 \%$ of all cases of acute ischemic stroke $(1,840)$, and only 2 patients presented with hemi-chorea and hemiballismus. Certainly, these involuntary movement disorders are unusual conditions in thalamic lacunar infarction compared with other common consequences such as pure sensory stroke, ataxic hemiparesis and hypesthetic ataxic hemiparesis [1-9]. In particular, choreiform movement is one of the rarest neurological symptoms of thalamic lacunar syndrome in current clinical practice [1-9]. Generally and classically, lacunar infarction in the subthalamic nucleus has been regarded as the lesion mainly responsible for hemi-chorea and hemi-ballismus, on the basis of previous anatomical studies [2,12,13,15]. Fisher [2] described delayed-onset hemi-dystonia in the distal part of the limbs as a common form of movement disorder following a focal lesion in the thalamus. However, D'Olhaberriague et al. [13] reported that hemi-chorea and hemi-ballismus were the most common types of movement disorders and the thalamus was the most frequently damaged cerebral structure. Dejerine and Roussy [1] were the first to report on a patient with a thalamic lesion with various neurological features as 
having the 'thalamic syndrome'. The typical thalamic syndrome is clinically characterized by persistent superficial hemianesthesia with deep sensory disturbance, recoverable mild ataxic hemiparesis with choreiform movements and severe spontaneous thalamic pain in the hypesthetic side [1]. In the thalamic syndrome, damage of proprioceptive sensation is crucially important for choreiform movements $[1,3-6,8]$. In previous studies, hemi-chorea or pseudochoreoathetosis, which is characterized by 'piano-playing' movements of the fingers on an outstretched hand, has been directly attributed to the proprioceptive sensory disturbance resulting from various lesions involving the sensory cortex, spinal cord, dorsal root ganglion or thalamus [1, 3-9]. However, our patient had no ataxic hemiparesis or sensory defects including proprioceptive sensory disturbance, and he developed sudden-onset right hemi-chorea as the only initial manifestation of left thalamic lacunar infarction. D'Olhaberriague et al. [13] described 2 patients who presented with hemi-chorea and hemi-ballismus without hemiparesis and hypesthesia caused by lateralized thalamic lacunar infarction. The right pure hemi-chorea resulting from an acute phase of left thalamic lacunar infarction in our patient may be similar to the pathological conditions of these 2 patients.

The mechanism responsible for the significantly rare and interesting neurological features observed in our patient is very difficult to speculate on in terms of its anatomical basis. At present, the most widely accepted hypothesis, which was proposed by DeLong [15], to explain hyperkinetic disorders such as chorea or ballismus highlights the loss of STN control on the internal segment of the GP (GPi), followed by the disinhibition of thalamic neurons. In this case, besides MRI findings, functional neuro-imaging was obtained by ${ }^{99 \mathrm{mTC}-E C D-S P E C T}$ as additional information. The remarkable hypoperfusion in the left thalamus on SPECT revealed a more extensive ischemic lesion than the acute lacunar infarction on the initial DWI. These findings suggest that the damage of cerebral autoregulation and the decline of neurofunctional activity affect the periphery of the left thalamus. The structural and functional damage in the periphery involving the thalamus suggests disruption in the various thalamic connecting fibers from the STN, GP, posterior limb of internal capsule and cerebellum, and consequently would lead to crucial derangement in the basal ganglia-cortical circuit. Therefore, it was considered that the crucial location of the thalamic peripheral damage is directly responsible for the pure hemi-chorea in this case. In addition, the relative $\mathrm{CBF}$ increasing in the left head of the caudate nucleus observed on simultaneous SPECT reflects the activation of neuronal function in the left striatum. This interesting phenomenon suggests that the striatal inhibitory effect toward the GP is strengthened. As a result, the degradation of GP, in particular GPi, activity would lead to the disinhibition of thalamic neurons. The authors considered that the abnormal thalamo-cortical neuronal inputs due to disinhibition from GPi control would advance the appearance of the pure hemi-chorea.

\section{Conclusion}

In this case, the left thalamic lesion due to acute lacunar infarction detected by an initial DWI was too small to cause major neuronal manifestations of the thalamic syndrome such as ataxic hemiparesis and sensory disturbances. In addition, it was speculated that the hypoperfusion in the periphery of the left thalamus and the 
hyperperfusion in the left head of the caudate nucleus observed on SPECT study suggested a fiber disruption and derangement in the basal ganglia-cortical circuit, and therefore these abnormal CBF alterations in the basal ganglia including thalamus and striatum would be responsible for the appearance of the contralateral pure hemichorea. However, it is still unknown why only a small proportion of patients with thalamic infarction develop such involuntary movements. The pathophysiological mechanism responsible for the choreiform movements following thalamic infarction is extremely difficult to understand and complicated; therefore, further research and an accumulation of cases are necessary to obtain a good understanding of this issue.

\section{Acknowledgement}

We are grateful to the patient for the permission to publish this information.

\section{Disclosure Statement}

The authors declare that they have no competing interests. There are no financial conflicts of interest in relation to this study. 

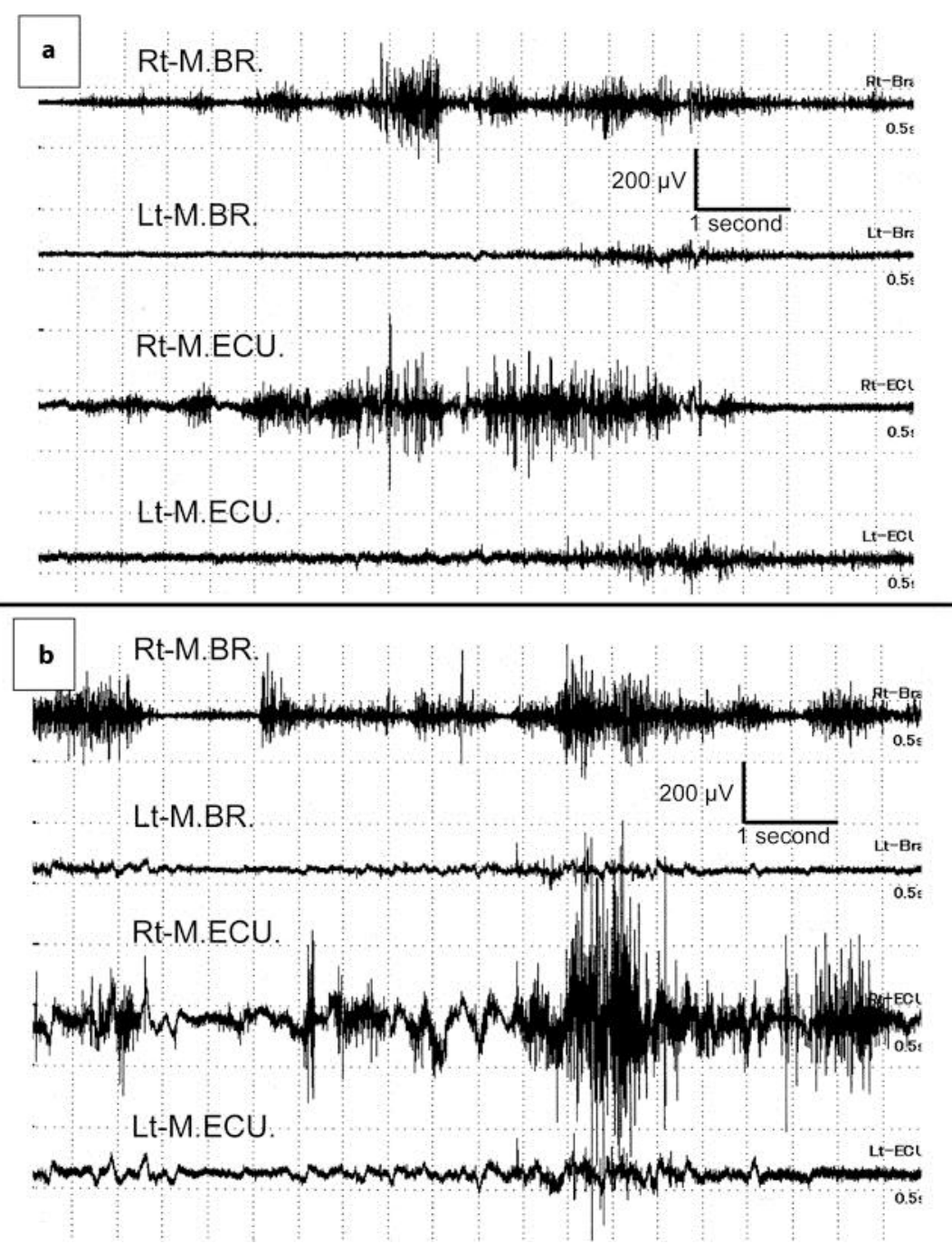

Fig. 1. sEMG recordings at rest (a) and under the calculation task (b). The sEMG recordings demonstrate several synchronized grouping discharges in the right musculus brachioradialis (M.BR.) and musculus extensor carpi ulnaris (M.ECU.). These grouping discharges appear irregularly and have a relatively long duration ( $>1 \mathrm{~s}$ ). In addition, the frequency and amplitude of the grouping discharges increase remarkably under the calculation task. Rt = Right; Lt = left. 

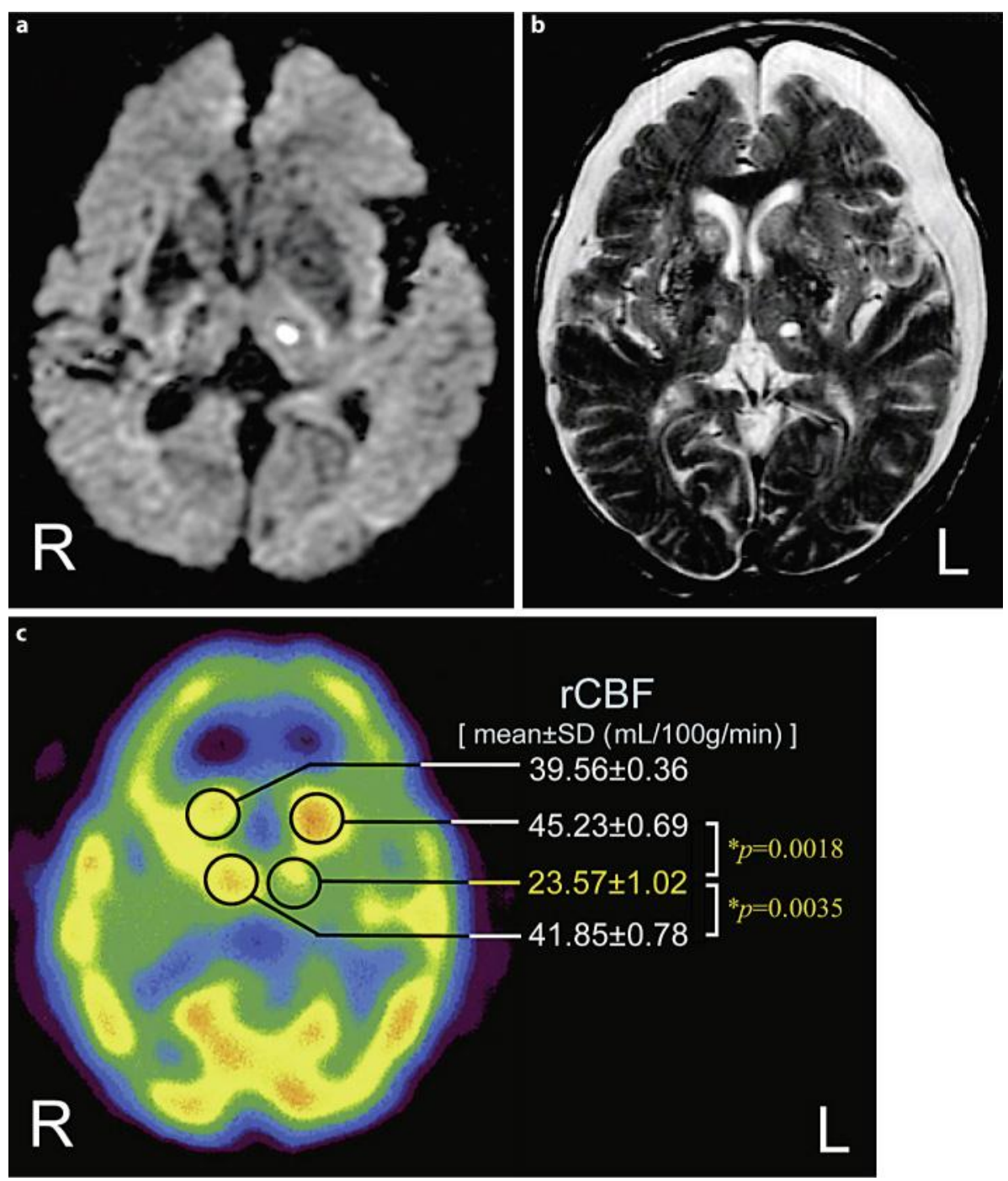

Fig. 2. The findings of neuro-imaging. The results of the MRI study are shown in a and $\mathbf{b}$. The 99mTcECD SPECT study result is shown in c (performed on day 18 after admission). a Axial section of the initial DWI (TR: 6,000, TE: 100), performed on admission. b Axial section of the follow-up T2-WI (TR: 4,500, TE: 90), performed on day 14 after admission. c Axial image of 99mTc-ECD-SPECT. The rCBF was quantified using a fully automated analysis software, FineSRT. The rCBF data are described as mean \pm SD (in milliliters per 100 grams per minute). A significant decline of rCBF in the left thalamus $(23.57 \pm 1.02)$ was demonstrated compared with those in the right $\left(41.85 \pm 0.78 ;{ }^{*} p=0.0035\right)$ and in the left head of the caudate nucleus $\left(45.23 \pm 0.69 ;{ }^{*} p=0.0018\right)$.

\section{References}

1 Dejerine J, Roussy G: Le syndrome thalamique. Rev Neurol (Paris) 1906;14:521-531.

-2 Fisher CM: Lacunar strokes and infarcts: a review. Neurology 1982;32:871-876.

-3 Pantano P, Di Cesare S, Ricci M, Gualdi GF, Sabatini U, Di Piero V: Hemichorea after a striatal ischemic lesion: evidence of thalamic disinhibition using single-photon emission computed tomography: a case report. Mov Disord 1996;11:444-447. 
4 Gille M, Van den Bergh P, Ghariani S, Guettat L, Delbecq J, Depre A: Delayed-onset hemidystonia and chorea following contralateral infarction of the posterolateral thalamus. A case report. Acta Neurol Belg 1996;96:307-311.

5 Lee MS, Kim YD, Kim JT, Lyoo CH: Abrupt onset of transient pseudochoreoathetosis associated with proprioceptive sensory loss as a result of a thalamic infarction. Mov Disord 1998;13:184-186.

6 Kim JW, Kim SH, Cha JK: Pseudochoreoathetosis in four patients with hypesthetic ataxic hemiparesis in a thalamic lesion. J Neurol 1999;246:1075-1079.

-7 Lera G, Scipioni O, Garcia S, Cammarota A, Fischbein G, Gershanik O: A combined pattern of movement disorders resulting from posterolateral thalamic lesions of a vascular nature: a syndrome with clinicoradiologic correlation. Mov Disord 2000;15:120-126.

-8 Tan EK, Chan LL, Auchus AP: Complex movement disorders following bilateral paramedian thalamic and bilateral cerebellar infarcts. Mov Disord 2001;16:968-970.

-9 Kim JS: Delayed onset mixed involuntary movements after thalamic stroke: clinical, radiological and pathophysiological findings. Brain 2001;124:299-309.

10 Chung SJ, Im JH, Lee MC, Kim JS: Hemichorea after stroke: clinical-radiological correlation. J Neurol 2004;251:725-729.

11 Galbreath AD, Goldstein LB: Dysnomia, ataxia, choreoathetosis, sensory impairment, and gait imbalance after lentiform nucleus stroke. J Stroke Cerebrovasc Dis 2009;18:494-496.

12 Park SY, Kim HJ, Cho YJ, Cho JY, Hong KS: Recurrent hemichorea following a single infarction in the contralateral subthalamic nucleus. Mov Disord 2009;24:617-618.

-13 D’Olhaberriague L, Arboix A, Martí-Vilalta JL, Moral A, Massons J: Movement disorders in ischemic stroke: clinical study of 22 patients. Eur J Neurol 1995;2:553-557.

-14 Arboix A, López-Grau M, Casasnovas C, García-Eroles L, Massons J, Balcells M: Clinical study of 39 patients with atypical lacunar syndrome. J Neurol Neurosurg Psychiatry 2006;77:381-384.

15 DeLong MR: Primate models of movement disorders of basal ganglia origin. Trends Neurosci 1990;13:281-285. 